PROCEEDINGS OF THE

AMERICAN MATHEMATICAL SOCIETY

Volume 127, Number 4, April 1999, Pages 1097-1102

S 0002-9939(99)04688-2

\title{
ON THE SCHWARZ SYMMETRY PRINCIPLE IN A MODEL CASE
}

\author{
JOËL MERKER AND FRANCINE MEYLAN
}

(Communicated by Steven R. Bell)

\begin{abstract}
In this article, we prove that smooth CR diffeomorphisms between two real analytic holomorphically nondegenerate hypersurfaces, one of which is rigid and polynomial, extend to be locally biholomorphic. It turns out that the result can be generalized to not totally degenerate mappings, in the sense of Baouendi and Rothschild.
\end{abstract}

\section{INTRODUCTION}

Since the fundamental work of Baouendi, Jacobowitz and Treves [BJT], no particular attention was given to the analog of the Schwarz symmetry principle in the complex euclidean space in the case of non essentially finite real analytic hypersurfaces, not to mention $[\mathrm{MEY}],[\mathrm{MM}]$. However, in view of the recent deep work of Baouendi, Huang and Rothschild [BHR], it can be easily conjectured that the local Schwarz symmetry principle holds for a $\mathcal{C}^{\infty}$-smooth $\mathrm{CR}$ diffeomorphism $f: M \rightarrow M^{\prime}$, between holomorphically nondegenerate real analytic hypersurfaces $M$ and $M^{\prime}$, which is holomorphic in one side of $M$, and that this is the optimal sufficient condition to get analyticity of a smooth CR mapping. In this paper, we give a short and elegant geometric proof of a precise and general statement in the case $M^{\prime}$ is polynomial and rigid. We do not assume that $M$ is algebraic, so our result does not follow from $[\mathrm{BHR}]$.

\section{Smooth CR Diffeomorphisms}

Let $M^{\prime}$ be a real analytic hypersurface in $\mathbb{C}^{n}$ and assume that its equation in coordinates $t=(w, z), t \in \mathbb{C}^{n}, w=\left(w_{1}, \ldots, w_{n-1}\right) \in \mathbb{C}^{n-1}, z \in \mathbb{C}$, is in the special form

$$
M^{\prime}: \quad \bar{z}=z+i \rho^{\prime}(w, \bar{w})=z+i \sum_{\alpha \in \mathbb{N}^{n-1},|\alpha| \leq N_{0}} \rho_{\alpha}^{\prime}(w) \bar{w}^{\alpha},
$$

where the function $\rho^{\prime}(w, \bar{w})$ is a polynomial in the variables $w, \bar{w}$, and $N_{0} \in \mathbb{N}$. Choose coordinates $t$ in $\mathbb{C}^{n}$ near $M$. Such an equation is usually called polynomial rigid. Then one has the following remarkable statement.

Received by the editors July 23, 1997.

1991 Mathematics Subject Classification. Primary 32H02; Secondary 32C05, 32C16.

Key words and phrases. Schwarz symmetry principle, CR mappings, real analytic manifolds.

The first author was partially supported by the École Normale Supérieure, and the second author by Swiss NSF Grant 2000-042054.94/1. 
Theorem 1.1. Let $M$ be a real analytic hypersurface in $\mathbb{C}^{n}$, let $p \in M$, let $f$ be a holomorphic mapping defined into a side $D$ of $M$ at $p, \mathcal{C}^{\infty}$ up to $M$, such that $f$ sends $(M, p) C R$ diffeomorphically into another real analytic hypersurface $\left(M^{\prime}, f(p)\right)$. Assume that there exist coordinates $(w, z)$ at $f(p)=0$ such that $M^{\prime}$ has a polynomial like equation as (1.1) and let $G(f, \lambda)$ denote $f_{n}+i \rho^{\prime}\left(f^{*}, \lambda\right), f^{*}=$ $\left(f_{1}, \ldots, f_{n-1}\right)$. Then

(a) $(t, \lambda) \mapsto G(f(t), \lambda)$ extends as a holomorphic function to $\mathcal{O}_{t}(0) \times \mathcal{O}_{\lambda}(0)$;

(b) In case the coordinates $(w, z)$ are normal, i.e. $\rho^{\prime}(w, 0) \equiv 0$, the normal component $f_{n}$ of $f$ extends as a holomorphic function to $\mathcal{O}_{t}(0)$;

(c) $f$ extends as a holomorphic function to $\mathcal{O}_{t}(0)$ if $M$ (hence $M^{\prime}$ too) is holomorphically nondegenerate.

Proof. Define the reflection function

$$
G(f(t), \lambda)=f_{n}(t)+i \rho^{\prime}\left(f^{*}(t), \lambda\right) .
$$

Now let $S \subset M$ be a real analytic totally real submanifold of dimension $n$ containing 0 . Since there exists $H$, a well-defined biholomorphism taking $S$ into a piece of $\mathbb{R}^{n}$ through 0, we can introduce an antiholomorphic reflection mapping $\sigma_{S},\left.\sigma_{S}\right|_{S}=\left.\mathrm{id}\right|_{S}$, by taking $\sigma_{S}(t):=H^{-1}(\overline{H(t)})$. Choose $\mathcal{W}^{-}$a wedge of edge $S$ near 0 such that $\mathcal{W}^{-} \subset D$ and $\sigma_{S}\left(\mathcal{W}^{-}\right)=: \mathcal{W}^{+} \subset U \backslash \bar{D}, U$ a neighborhood of 0 .

First, we notice that $G(f(t), \lambda)$ is holomorphic over $D \times \mathcal{O}_{\lambda}(0)$ and $\mathcal{C}^{\infty}$ over $(D \cup M) \times \mathcal{O}_{\lambda}(0)$. By the assumption that $f(M) \subset M^{\prime}$, we have

$$
\overline{f_{n}(t)}=G\left(f(t), \overline{f^{*}(t)}\right), \quad \text { for } t \in M \text {. }
$$

Choose a basis $\left\{L_{1}, \ldots, L_{n-1}\right\}$ of the complex tangent bundle $T^{1,0} M$ with analytic coefficients in $(t, \bar{t})$. Applying $\bar{L}_{j}$ to the previous equation, one gets:

$$
\bar{L}_{j} \overline{f_{n}(t)}=\sum_{k=1}^{n-1} \frac{\partial G}{\partial \lambda_{k}} \bar{L}_{j} \bar{f}_{k} .
$$

Let $\mathcal{J}$ denote the matrix $\left(\bar{L}_{j} \bar{f}_{k}\right)_{1 \leq j, k \leq n-1}$ and set $J=\operatorname{det} \mathcal{J}$. Since $f$ is a CR diffeomorphism, $J(t) \neq 0$ for $t \in M$. We now have

$$
\left(\frac{\partial G}{\partial \lambda_{1}}, \ldots, \frac{\partial G}{\partial \lambda_{n-1}}\right)^{\tau}=\mathcal{J}^{-1}\left(\overline{L f^{*}}\right)\left(\bar{L}_{1} \overline{f_{n}}, \ldots, \bar{L}_{n-1} \overline{f_{n}}\right)^{\tau}
$$

( $\tau$ denotes transposition). Writing $(1.3)$ as $(n-1)$ scalar equations, applying $\bar{L}_{j}$ to each of them and proceeding in this manner, we see, by induction, that for each multiindex $\beta=\left(\beta_{1}, \ldots, \beta_{n-1}\right)$, there are two holomorphic functions $P_{\beta}^{(1)}$ and $P_{\beta}^{(2)}$ in the arguments $\left(t, \bar{t},\left\{\bar{L}^{\gamma} \bar{f}\right\}_{|\gamma| \leq|\beta|}\right)$ and $\left(t, \bar{t},\left\{\bar{L}^{\gamma} \bar{f}\right\}_{|\gamma| \leq 1}\right)$ such that, for each $t \in M$, one has $P_{\beta}^{(2)}\left(t, \bar{t},\left\{\bar{L}^{\gamma} \bar{f}\right\}_{|\gamma| \leq 1}\right) \neq 0$ and

$$
\partial_{\lambda}^{\beta} G\left(f(t), \overline{f^{*}(t)}\right)=\frac{P_{\beta}^{(1)}\left(t, \bar{t},\left\{\bar{L}^{\gamma} \bar{f}\right\}_{|\gamma| \leq|\beta|}\right)}{P_{\beta}^{(2)}\left(t, \bar{t},\left\{\bar{L}^{\gamma} \bar{f}\right\}_{|\gamma| \leq 1}\right)} .
$$

Here, $L^{\gamma}$ denotes $L_{1}^{\gamma_{1}} \cdots L_{n-1}^{\gamma_{n-1}}$. Since $P_{\beta}^{(2)}$ does not vanish on $M$, we see that the function $P_{\beta}^{(1)} / P_{\beta}^{(2)}$ has a continuous extension to $M$, which we will denote by $h_{\beta}\left(t, \bar{t},\left\{\bar{L}^{\gamma} \bar{f}\right\}_{|\gamma| \leq|\beta|}\right)$. Recall that since $\rho^{\prime}$ is a polynomial, $\partial_{\lambda}^{\beta} G$ becomes zero for $|\beta|$ sufficiently large, say $|\beta| \geq N_{0}+1$. Set, for $t \in \mathcal{W}^{+},|\beta| \leq N_{0}, \tilde{h}_{\beta}(t):=$ 
$h_{\beta}\left(t, \overline{\sigma_{S}(t)},\left\{\bar{L}^{\gamma} \overline{f\left(\sigma_{S}(t)\right)}\right\}_{|\gamma| \leq|\beta|}\right)$. Since $t \mapsto \sigma_{S}(t)$ is antiholomorphic in $t, \tilde{h}_{\beta}$ extends as a holomorphic function into $\mathcal{W}^{+}$and continuous in $\mathcal{W}^{+} \cup S$. Now let

$$
\begin{array}{rlrl}
\phi_{\beta}^{+}:=\frac{1}{\beta !}\left(\partial_{\lambda}^{\beta} \sum_{|\gamma| \leq N_{0}} \tilde{h}_{\gamma}(t)\left(\lambda-\overline{f^{*}\left(\sigma_{S}(t)\right)}\right)^{\gamma}\right)_{\lambda=0}, & & \text { for } t \in \mathcal{W}^{+},|\beta| \leq N_{0}, \\
\phi_{\beta}^{-}(t):=\frac{1}{\beta !}\left(\partial_{\lambda}^{\beta} G(f(t), \lambda)\right)_{\lambda=0}, & \text { for } t \in \mathcal{W}^{-},|\beta| \leq N_{0} .
\end{array}
$$

Notice that $\phi_{\beta}^{+}$matches up with $\phi_{\beta}^{-}$over $S$, by (1.4). Then the edge of the wedge theorem implies that there exists a neighborhood $V$ of 0 such that each function equal to $\phi_{\beta}^{-}$in $\mathcal{W}^{-}, \phi_{\beta}^{+}$in $\mathcal{W}^{+}$, extends as a holomorphic function $\phi_{\beta}$ defined in $V$, which can be filled in by analytic discs with boundaries in $\mathcal{W}^{+} \cup \mathcal{W}^{-}$. Now,

$$
\sum_{|\beta| \leq N_{0}} \phi_{\beta}(t) \lambda^{\beta}
$$

clearly gives the desired holomorphic extension for $G(f(t), \lambda)$ to $\mathcal{O}_{t}(0) \times \mathcal{O}_{\lambda}(0)$. The proof of (a) is complete.

If coordinates $(w, z)$ are normal, the relation $G(f(t), 0)=f_{n}(t)=\phi_{0}(t)$ shows that the normal component of $f$ extends holomorphically at 0 . This gives (b).

Since $M^{\prime}$ is holomorphically nondegenerate, the complex analytic set at 0

$$
\Delta^{\prime}=\left\{(w, z) \in \mathbb{C}^{n} ; \operatorname{det}\left(\frac{\partial \rho_{\alpha_{j}}^{\prime}}{\partial w_{j}}\right)_{1 \leq i, j \leq n-1}(w)=0, \quad \forall\left(\alpha_{1}, \ldots, \alpha_{n-1}\right) \in \mathbb{N}^{(n-1)^{2}}\right\}
$$

has complex codimension greater than one [S]. Hence $\mathbb{C}^{n} \backslash \Delta^{\prime}$ is connected, and $M^{\prime} \backslash\left(M^{\prime} \cap \Delta^{\prime}\right)$ too, since $T_{0} M^{\prime}=\{\bar{z}=z\}$ and the equations of $\Delta^{\prime}$ depend only on the $w$-coordinate. Set

$$
\mathcal{C}=\left\{(t, \lambda, \mu) \in \mathbb{C}^{n} \times \mathbb{C}^{n} ; t \in M, \mu=\phi_{0}(t), \rho_{\alpha}^{\prime}(\lambda)=\rho_{\alpha}^{\prime}\left(f^{*}(t)\right), \forall|\alpha| \geq 1\right\} .
$$

According to (b), $\mathcal{C}$ is a real analytic subset of $\mathbb{C}^{n}$. Furthermore, $\mathcal{C}$ contains the germ at 0 of a $\mathcal{C}^{\infty}$ smooth $(2 n-1)$-dimensional manifold, namely the graph of $f$,

$$
\Gamma=\left\{(t, f(t)) \in \mathbb{C}^{n} \times \mathbb{C}^{n} ; t \in M\right\} .
$$

Lemma 1.2. For $p \in M$, if $f(p) \in M^{\prime} \backslash\left(M^{\prime} \cap \Delta^{\prime}\right)$, then $\mathcal{C} \equiv \Gamma$ in a neighborhood of $(p, f(p))$.

Proof. Apply the implicit function theorem.

Since $M \backslash\left(f^{-1}\left(M^{\prime} \cap \Delta^{\prime}\right)\right)$ is connected too (recall $f$ is a diffeomorphism), Lemma 1.2 implies that a single irreducible component $\mathcal{C}_{1}$ of the real analytic set $\mathcal{C}$ contains $\Gamma, \operatorname{dim}_{\mathbb{R}} \mathcal{C}_{1}=2 n-1$. To conclude that $f$ is analytic (hence holomorphically extendable, by complexification), apply the following theorem of Malgrange: $A \mathcal{C}^{\infty}$-smooth $q$-dimensional $(q \geq 1)$ manifold contained in a real analytic set of dimension $q$ is a real analytic manifold $[\mathrm{BHR}]$.

Remark 1.3. Under the hypothesis of (c), $M^{\prime}$ being rigid and nondegenerate cannot contain a complex hypersurface through $f(p)$, so $M$ is minimal too and we do not have to assume that $f$ is holomorphic in one side of $M$ at $p$, because of Trépreau's extension theorem. 
Remark 1.4. The authors conjecture that statements (a), (b) are true for any mapping $f$ between real analytic hypersurfaces without assuming that $M^{\prime}$ is polynomial rigid nor that $\operatorname{det} J_{f}(p) \neq 0$.

\section{Not totally DEGEnERATE CR MAPPINGS}

Theorem 1.1 is easily seen to be true if one assumes only that the mapping $f$ is not totally degenerate in the sense that $\operatorname{det}\left(\frac{\partial F_{i}}{\partial w_{j}}\right)(w, 0) \not \equiv 0$, where $F_{1}, \ldots, F_{n-1}$ denote the formal power series of $f_{1}, \ldots, f_{n-1}$ at 0 . We shall get the following refinement of Theorem 1.1 (cf. [MIR]).

Theorem 2.1. (a), (b) and (c) are valid for $\mathcal{C}^{\infty} f$ not totally degenerate.

Proof. Let $\tilde{\mathcal{J}}$ denote the adjoint matrix of $\mathcal{J}=\left(\bar{L}_{j} \bar{f}_{k}\right)_{1 \leq j, k \leq n-1}$, so that $\tilde{\mathcal{J}} \mathcal{J}=$ $\operatorname{det} \mathcal{J} \mathrm{Id}=J$ Id. Applying $\bar{L}_{j}$ to the fundamental equation $\bar{f}_{n}=G\left(f, \bar{f}^{*}\right)$, we get

$$
J\left(\partial_{\lambda_{k}} G\left(f, \bar{f}^{*}\right)\right)_{1 \leq k \leq n-1}=\tilde{\mathcal{J}}\left(\bar{L}_{1} \bar{f}_{n}, \ldots, \bar{L}_{n-1} \bar{f}_{n}\right)^{\tau} .
$$

Assume, by induction, that for each $\beta \in \mathbb{N}^{n-1}$ with $|\beta| \leq k_{0}$, there exists a holomorphic polynomial $g_{\beta}$ such that, on $M$,

$$
J^{2|\beta|-1} \partial_{\lambda}^{\beta} G\left(f, \bar{f}^{*}\right)=g_{\beta}\left(t, \bar{t},\left\{\bar{L}^{\gamma} \bar{f}\right\}_{|\gamma| \leq|\beta|}\right) .
$$

Prove it for $|\beta|=k_{0}+1$. Indeed, applying $\bar{L}_{j}, j=1, \ldots, n-1$, to (2.1), we get

$$
\begin{aligned}
(2|\beta|-1) J^{2|\beta|-2} \bar{L}_{j}(J) \partial_{\lambda}^{\beta} G\left(f, \bar{f}^{*}\right)+J^{2|\beta|-1} & \sum_{k=1}^{n-1} \partial_{\lambda_{k}} \partial_{\lambda}^{\beta} G\left(f, \bar{f}^{*}\right) \bar{L}_{j} \bar{f}_{k} \\
& =g_{j \beta}\left(t, \bar{t},\left\{\bar{L}^{\gamma} \bar{f}\right\}_{|\gamma| \leq|\beta|+1}\right),
\end{aligned}
$$

where the $g_{j \beta}$ are holomorphic polynomials. Multiplying the equation by $J$, and replacing $J^{2|\beta|-1} \partial_{\lambda}^{\beta} G\left(f, \bar{f}^{*}\right)$ by its value given by $(2.1)$, we get

$$
J^{2|\beta|} \sum_{k=1}^{n-1} \partial_{\lambda_{k}} \partial_{\lambda}^{\beta} G\left(f, \bar{f}^{*}\right) \bar{L}_{j} \bar{f}_{k}=g_{j \beta}^{\prime}\left(t, \bar{t},\left\{\bar{L}^{\gamma} \bar{f}\right\}_{|\gamma| \leq|\beta|+1}\right), \quad j=1, \ldots, n-1,
$$

for some polynomial $g_{j \beta}^{\prime}$. Then (2.1) follows at order $k_{0}+1$ by multiplying (2.2) by $\tilde{\mathcal{J}}$. Recall that since $G$ is a polynomial, the equations $(2.1)$ are $0=0$, i.e. $\partial_{\lambda}^{\beta} G \equiv 0$, for $|\beta| \geq N_{0}+1$. Now, according to [BR], there exists $\gamma \in \mathbb{N}^{n-1}$ such that $\bar{L}^{\gamma} J(0) \neq 0$, and thus also, for each $\beta$, there exists $\gamma=\gamma(|\beta|)$ such that $\left(\bar{L}^{\gamma} J^{2|\beta|-1}\right)(0) \neq 0$. This implies that for each $\beta$ with $|\beta|=N_{0}$, we have

$$
\partial_{\lambda}^{\beta} G\left(f, \bar{f}^{*}\right)=\partial_{\lambda}^{\beta} G(f, 0)=\frac{\bar{L}^{\gamma}\left(g_{\beta}\left(t, \bar{t},\left\{\bar{L}^{\gamma} \bar{f}\right\}_{|\gamma| \leq|\beta|}\right)\right)}{\bar{L}^{\gamma}\left(J^{2|\beta|-1}\right)}=h_{\beta}\left(t, \bar{t},\left\{\bar{L}^{\gamma} \bar{f}\right\}_{|\gamma| \leq \Gamma(\beta)}\right),
$$

for some holomorphic function $h_{\beta}$ near 0 and $\Gamma(\beta) \geq \sup \left\{\gamma(|\beta|) ;|\beta|=N_{0}\right\}+N_{0}$. Assume by downards induction that, for each $k_{0}+1 \leq|\beta| \leq N_{0}$, there exists a holomorphic function $h_{\beta}$ on a neighborhood of 0 in $\mathbb{C}^{\Gamma(\beta)+2 n}$ such that $(2.3)$ is true on $M$. Prove it for $|\beta|=k_{0}$. In fact, there exists a holomorphic polynomial $d_{\beta}$ such that

$$
\left(\partial_{\lambda}^{\beta} G\right)\left(f, \bar{f}^{*}\right)=\left(\partial_{\lambda}^{\beta} G\right)(f, 0)+d_{\beta}\left(\left\{\left(\partial_{\lambda}^{\beta} G\right)(f, 0)\right\}_{k_{0}+1 \leq|\beta| \leq N_{0}}, \bar{f}^{*}\right),
$$


so that, by (2.1) and (2.3), we can write

$$
\begin{aligned}
& J^{2|\beta|-1}\left(\partial_{\lambda}^{\beta} G\right)(f, 0)=g_{\beta}\left(t, \bar{t},\left\{\bar{L}^{\gamma} \bar{f}\right\}_{|\gamma| \leq|\beta|}\right)- \\
& J^{2|\beta|-1} d_{\beta}\left(\left\{h_{\beta}\left(t, \bar{t},\left\{\bar{L}^{\gamma} \bar{f}\right\}_{|\gamma| \leq \Gamma(\beta)}\right\}_{k_{0}+1 \leq|\beta| \leq N_{0}}, \bar{f}^{*}\right),\right.
\end{aligned}
$$

and (2.3) follows by applying some power of $\bar{L}$ to (2.4). Since we obtained the representation (2.3), (a) and (b) follow along the lines of 1.1. (The above calculations are extracted from $[\mathrm{BR}]$.)

To prove (c), it is sufficient to show that the set

$$
\Delta=\left\{(w, z) \in M ; \operatorname{det}\left(L_{j} f_{k}\right)_{1 \leq j, k \leq n-1}(w, z)=0\right\}
$$

is of Hausdorff codimension at least two, so that $M \backslash \Delta$ is connected. Indeed, let $U \subset M \backslash \Delta$ be a small open set such that $f: U \rightarrow f(U)=V$ is CR isomorphic. Then $U \backslash f^{-1}\left(V \cap \Delta^{\prime}\right)$ is connected too, so $M_{1}=(M \backslash \Delta) /\left(f^{-1}\left(\Delta^{\prime} \cap f(M \backslash \Delta)\right)\right)$ is connected too and Lemma 1.2 shows that $M_{1} \equiv \mathcal{C}$ there.

Lemma 2.2. The set $\Delta \subset M$ is of Hausdorff dimension $\leq 2 n-3$ in a neighborhood of the origin.

Proof. Let $\delta$ denote the $\mathcal{C}^{\infty}$-smooth function on $M, \delta=\operatorname{det}\left(L_{j} f_{k}\right)_{1 \leq j, k \leq n-1}$. Since $f$ is not totally degenerate at 0 , we can write (recall $\delta$ is flat in $\bar{w}$ at 0 )

$$
\delta(w, \bar{w}, x)=P(w)+\sum_{|\beta|=N+1} w^{\beta} R_{\beta}(w, \bar{w}, x)+x Q(w, \bar{w}, x),
$$

for some nonzero polynomial $P$ and $\mathcal{C}^{\infty}$ functions $R_{\beta}, Q$. We study the zero locus of $\delta$. We can assume that $\delta\left(w_{1}, 0, \bar{w}_{1}, 0,0\right)=w_{1}^{N}\left(1+S\left(w_{1}, \bar{w}_{1}\right)\right)$, with $S$ $\mathcal{C}^{\infty}$, flat in $\bar{w}_{1}$ at $0, S(0)=0$. Malgrange's preparation theorem yields that there exist $\mathcal{C}^{\infty}$ functions $q$ and $r$ on $M$ with $q(0) \neq 0$ and $r(0) \neq 0$ such that (writing $\left.w^{*}=\left(w_{2}, \ldots, w_{n-1}\right)\right)$

$$
(q \delta)(w, \bar{w}, x)=u_{1}^{N}+\sum_{1 \leq j \leq N} \lambda_{j}\left(v_{1}, w^{*}, \bar{w}^{*}, x\right) u_{1}^{N-j}
$$

and

$$
(r \delta)(w, \bar{w}, x)=v_{1}^{N}+\sum_{1 \leq j \leq N} \mu_{j}\left(u_{1}, w^{*}, \bar{w}^{*}, x\right) v_{1}^{N-j},
$$

for $\mathcal{C}^{\infty}$ functions $\lambda_{j}, \mu_{j}, 1 \leq j \leq N$, vanishing at 0 . Fixing small $\left(w^{*}, \bar{w}^{*}, x\right)$, one sees that there can be at most $N^{2}$ solutions to the system of the two equations above.

\section{REFERENCES}

[BJT] S. Baouendi, H. Jacobowitz and F. Treves, On the real analyticity of CR mappings, Ann. Math. 122 (1985), 365-400. MR 87f:32044

[BR] S. Baouendi and L. Rothschild,, Germs of CR maps between hypersurfaces in complex space, Invent. Math. 93 (1988), 481-500. MR 90a:32036

[BHR] S. Baouendi, X. Huang and L. Rothschild, Regularity of CR mappings between algebraic hypersurfaces, Invent. Math. 125 (1996), 13-36.

[MER] J. Merker, On the Schwarz symmetry principle in three-dimensional complex euclidean space, Publications du Laboratoire de Mathématiques de l'École Normale Supérieure, Paris, Juillet 1997. Available at: http://www.dmi.ems.fr/edition/preprints

[MEY] F. Meylan, A reflection principle un complex space for a class of hypersurfaces and mappings, Pacific Math. J. 169 (1995), 135-160. MR 96f:32018 
[MM] F. Meylan and H.-M. Maire, Extension of smooth CR mappings between non essentially finite hypersurfaces in $\mathbb{C}^{3}$, Ark. Mat. 35 (1997), 185-199. CMP 97:11

[MIR] N. Mir, An algebraic characterization of holomorphic degeneracy and analyticity of CR mappings, Preprint, 1997.

[S] N. Stanton, Infinitesimal CR automorphisms of real hypersurfaces, Amer. J. Math. 118 (1996), 209-233. MR 97h:32027

Centre de Mathématiques et d'Informatique, Laboratoire D'Analyse, Topologie et Probabilités, 39 rue Joliot Curie, F-13453 Marseille Cedex 13, France

E-mail address: merker@dmi.ens.fr

E-mail address: merker@gyptis.univ-mrs.fr

Université de Fribourg, Institut de Mathématiques, 1700 Perolles, Fribourg, Suisse

E-mail address: meylan@unifr.ch 